\title{
Compression of finite size polymer brushes
}

\author{
T.A. Vilgis*, A. Johner and J.-F. Joanny \\ Institut Charles Sadron (UPR CNRS 022) \\ 6 rue Boussingault, 67083 Strasbourg Cedex, France \\ L.E.A MPI Mainz/ICS Strasbourg
}

\begin{abstract}
We consider edge effects in grafted polymer layers under compression. For a semi-infinite brush, the penetration depth of edge effects $\xi \propto h_{0}\left(h_{0} / h\right)^{1 / 2}$ is larger than the natural height $h_{0}$ and the actual height $h$. For a brush of finite lateral size $S$ (width of a stripe or radius of a disk), the lateral extension $u_{S}$ of the border chains follows the scaling law $u_{S}=\xi \varphi(S / \xi)$. The scaling function $\varphi(x)$ is estimated within the framework of a local Flory theory for stripe-shaped grafting surfaces. For small $x, \varphi(x)$ decays as a power law in agreement with simple arguments. The effective line tension and the variation with compression height of the force applied on the brush are also calculated.
\end{abstract}

PACS: 36.20.Ey, 42.70.Jk

\section{INTRODUCTION}

Grafted polymer layers are widely used to prevent colloidal aggregation, and therefore stabilize colloidal solutions. They are also utilized to modify the mechanical properties of solid - solid contacts [1]. They have attracted considerable theoretical attention [2,3] since the pioneering work by Alexander and de Gennes [⿶, F]. The overall thickness of the layer results from a balance between the stretching energy of the chains and the excluded volume interaction as shown in fig. (1).

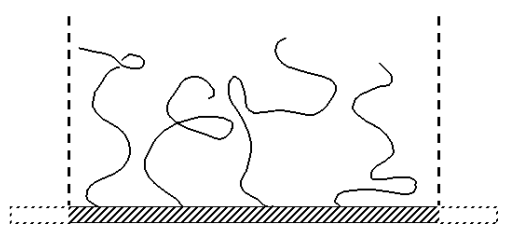

FIG. 1. A portion of an infinite brush : The chains stretch away from the grafting surface to lower their excluded volume interaction.

Most of the work, however, has been devoted to infinite brushes. In this case a Flory theory or a blob argument can be easily formulated to calculate the properties of the brush, i.e., its equilibrium height and its free energy. In a Flory-type approach, the natural thickness $h_{0}$ of a brush composed of linear chains of contour length $N$ at grafting density $\sigma$ minimizes the free energy per unit area that can be written as:

$$
F=k_{\mathrm{B}} T\left(\sigma h^{2} / N a^{2}+\frac{v}{2}(\sigma N / h)^{2} h\right),
$$

where the first term stands for the elastic energy and the second for the excluded volume energy. In a good solvent, the Edwards excluded volume parameter $v$ is positive. The minimization with respect to the height $h$ leads to the equilibrium height $h_{0}$ and the equilibrium free energy $F_{0}$

$$
h_{0}^{3}=v N^{3} \sigma a^{2} / 4 \quad \text { and } \quad F_{0} / k_{\mathrm{B}} T=3 \sigma N\left(\frac{\sigma v}{4 a}\right)^{2 / 3} .
$$

When the brush is compressed down to a height $h<h_{0}$, the excluded volume term dominates over the elastic term. The Flory-type free energy per unit area $F_{\infty}$ can then be rewritten in order to emphasize the ratio of the excluded volume to the elastic energy. It is convenient for the rest of the paper to introduce a correlation length $\xi$ and to write the free energy of the brush with infinite extension (no edge effects)

$$
F_{\infty}=k_{\mathrm{B}} T \frac{\sigma h^{2}}{N a^{2}}\left(1+\frac{\xi^{2}}{4 h^{2}}\right)
$$

The length $\xi$ defined by $\xi^{2}=8 h_{0}^{3} / h$, is larger than both the actual height $h$ and the natural height $h_{0}$. Although the definition of $\xi$ might not be obvious at present, it will turn out below that it plays a major role in the description of edge effects, due to the fact that the chains have more degrees of freedom close to the border of the brush than in the center.

Earlier, Raphaël and de Gennes proposed a description of the edge of a unconstrained semi-infinite brush that captures the essential physics [6]. They describe the edge effects on the free energy of the layer by a polarization $P$ proportional to the extension of the chains parallel to the grafting plane. The elastic energy contribution due to splay is then $\propto P^{2}$ and the next relevant term in a Landau expansion accounting for non uniform

*Permanent address: Max-Planck-Institute for Polymer Research, Ackermannweg 10, 55128 Mainz, Germany 
splay is therefore $\propto(\nabla P)^{2}$. The problem contains the unique length scale $h_{0}$ and the splay at the edge entails a negative effective line tension $\tau \sim-F_{0} h_{0}$.

A more refined description of polymer brushes originating in an idea by Semenov [7] was proposed and developed over the last decade [8,3]. This takes advantage of the drastic reduction of chain path fluctuations due to chain tension. Taking into account only most probable paths the model is exactly solvable, it gives access to the free end density and predicts the concentration profile. When the chain ends are constrained to the very edge of the layer, a situation similar to the Alexander model is recovered.

Several models have also been proposed in the case where the chain paths are not parallel straight lines [9, 10]. A major difficulty arises when the minimal paths are curved : it is then obvious that there is not a unique minimal path through a given point. To overcome this difficulty, either the free end points have been constrained (fixed) on the outer surface of the layer following Frederickson and coworkers [9] or a unique path description is used in a locally parabolic molecular potential scheme [10]. Both models can be solved for weakly disturbed brushes. Milner's model seems very successful to estimate free energies as illustrated by the calculation of block copolymer phase diagrams. Those models can be looked at as variational approaches. As we will be concerned with finite to huge deviations from the unconstrained brush we propose here a simpler model where the chain trajectories are straight lines and the free energy is calculated in a local Flory theory. In a sense this is a crude version of the previous models and in line with the scaling arguments used later on. The close link to the Raphaël - de Gennes approach will also appear clearly.

The paper is organized as follows. We first introduce and describe our simple model. It is then solved in the case of a semi-infinite brush. In section III we consider finite brushes before discussing the relevance of the present work to some experiments.

\section{THE MODEL AND THE SEMI-INFINITE BRUSH CASE}

In this section we introduce the local Flory model and compute the edge effects in a semi - infinite brush. This is a grafted polymer layer which has only one edge, whereas in the other direction the grafting surface is infinite. This case is useful to find the appropriate length scales. Throughout the paper we consider the chain trajectories to be straight lines. A chain starting at the grafting surface fills up a volume delimited by the nearby chains and the confining surface. The free energy is then a functional of the splay.

To be more specific, let us consider a semi-infinite brush grafted on the half-plane $x<0$ with a uniform grafting density $\sigma$ and let the (large) length along the $y$-axis be $L_{y}$. A chain starting at x, ends on the opposite surface at $X(x)$ shows a splay $u(x)=X-x$ and fills a box of volume $h \sigma^{-1}\left(1+u^{\prime} / 2\right)$, with $u^{\prime}=d u / d x$ as shown in Figure 2.
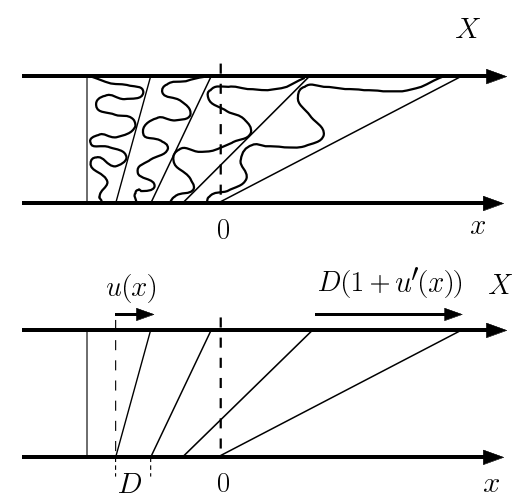

FIG. 2. The box model : A chain path is pictured as a straight line defined by the grafting coordinate $x$ and the splay $\mathrm{u}(\mathrm{x})$, the chain occupies a volume defined by the nearby chains.

The free energy functional reads

$$
\begin{gathered}
F / k_{\mathrm{B}} T=\frac{L_{y}}{N a^{2}} \sigma \int_{-\infty}^{0} d x\left[u(x)^{2}+h^{2}\right]+ \\
v L_{y} \frac{N^{2} \sigma^{2}}{h} \int_{-\infty}^{0} d x \frac{1}{2+u^{\prime}} .
\end{gathered}
$$

The Euler Lagrange equation obtained after functional minimization of eq. (㺼 with respect to the splay $u(x)$ has the first integral,

$$
u^{2}+\xi^{2} \frac{1+u^{\prime}}{\left(2+u^{\prime}\right)^{2}}=C,
$$

where the characteristic length scale $\xi$ has been introduced previously. Note that $\xi$ is much larger than both $h$ and $h_{0}$ at high compression. The integration constant $C$ is imposed by the boundary conditions. In the case of the semi-infinite brush, where the splay $u$ and the deformation $u^{\prime}$ vanish at $x=-\infty$, the appropriate choice is $C=1 / 4 \xi^{2}$. It is useful to introduce the dimensionless variables $\tilde{u}=u / \xi$ and $\tilde{x}=x / \xi$. The integration of eq.(5) shows that the splay tail decays exponentially over a characteristic length $\xi / 4$,

$$
\log \frac{\tilde{u}}{\tilde{u}_{0}}-2\left(\tilde{u}-\tilde{u}_{0}\right)=4 \tilde{x}
$$

The splay $u_{0}$ towards the very edge remains to be determined. This is done by calculating the effective line tension (i.e., the (negative) free energy cost of the splay 
per unit length of the border line) and minimizing ${ }^{1}$ it with respect to $\tilde{u}_{0}$. We obtain $\tilde{u}_{0}=1 / 2$ and the effective line tension :

$$
\tau=-\frac{k_{\mathrm{B}} T}{48} \frac{\sigma}{N a^{2}} \xi^{3} .
$$

As expected the line tension is negative as the splay relaxes some pressure at the brush edge. In fact, $\tau$ represents a fraction of the energy stored in the edge of size $\sim \xi$. Each chain in the edge contributes a in-plane tension $\tau_{\mathrm{i}} \sim k_{\mathrm{B}} T \xi / N a^{2}$ and at the level of scaling arguments, the in-plane tensions compensate the osmotic pressure inside the brush.

The penetration depth $\xi$ has a simple physical meaning. The elastic energy of a chain at the edge $U \sim$ $\xi^{2} / a^{2} N$ compensates the excluded volume energy of a bulk chain. Alternatively, the chains over the penetration length $\xi$ build up a brush almost parallel to the grafting surface with an effective grafting density $\sigma_{\|} \sim \sigma \xi / h$ with a brush "height" $\xi$, as shown in Figure 3.

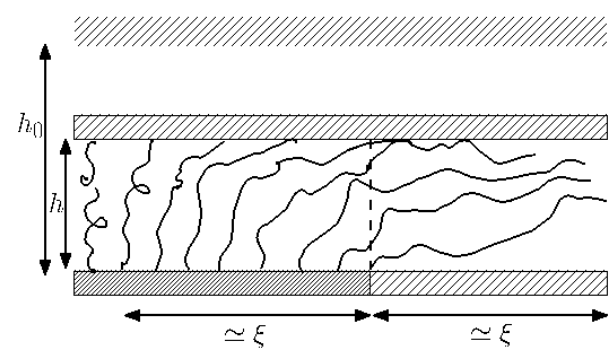

FIG. 3. The penetration depth $\xi$ of the edge effect : The chains located within the penetration depth build a brush parallel to the grafting surface at density $\sim \sigma \xi / h$ per lateral unit area that extends over a width $\xi$.

When calculating the excluded volume free energy in eq.(A), we have assumed a continuous distribution of chains i.e. $\xi / D>>1$, with $D=\sigma^{-1 / 2}$ the distance between neighboring grafted chains, which is fair. For $d u / d x<<1$ we could further expand the excluded volume energy, the first relevant term is then $\propto(\nabla u)^{2}$ similar to the square gradient term of Raphaël and de Gennes mentioned above [6]. The final minimization of the line tension with respect to the splay at the edge introduces a weakly singular solution at the border (the derivative $d u / d z$ diverges there in order to ensure a vanishing concentration) that is however of no serious consequence. The present very simple model forces the boxes to be filled up with monomers. This assumption is reasonable as long as the polymer "pushes" on the surfaces but obviously fails at the very edge where the chain boxes are very large and we nevertheless assume that the chain stretch over the largest box size. The model also allows for half of the available space only to be occupied. At the level of scaling arguments our approach is sound, however we cannot expect very accurate prefactors or scaling functions. A better model allows the chain to choose how much of the box height it wants to occupy. Let $H$ be the box height occupied by the chain, the volume occupied by the chain becomes $H \sigma^{-1}\left(1+u^{\prime} H / 2 h\right), H$ minimizes the chain chemical potential, this links $H$ to the splay derivative:

$$
\left(\frac{H}{4 h_{0}}\right)^{3}=\frac{1+u^{\prime} H / h}{\left(2+u^{\prime} H / h\right)^{2}\left(1+u^{2} / h^{2}\right)} .
$$

The simple model with filled boxes is valid as long as $H>h$ i.e. using eq.(8) for values of the splay up to $\xi / \sqrt{8}$ for strongly compressed brushes and for a vanishing splay in an uncompressed brush as expected (though $u_{0} \sim h_{0}$ remains obviously qualitatively correct for the uncompressed brush). In the remainder we use the simple box model in combination with scaling laws.

As shown above, chains over the penetration depth $\xi$ form a brush parallel to the grafting surface of "height" $\xi$. This merely states that there is a unique length scale governing the edge effects. Assuming this to remain true when the chains follow the excluded volume statistics where $h_{0} \sim N \sigma^{\frac{1-\nu}{\nu(d-1)}}$, we obtain the scaling behavior:

$$
\xi \sim h_{0}\left(\frac{h_{0}}{h}\right)^{\frac{1-\nu}{\nu d-1}}
$$

with $\nu$ the swelling exponent and $d$ the space dimension. The splay relaxes the constraint over the edge where a finite fraction of the free energy is saved, this corresponds to an effective line tension:

$$
\tau \sim \tau_{0}\left(\frac{h_{0}}{h}\right)^{\frac{2-\nu}{d \nu-1}}
$$

where $\tau_{0} \sim-F_{0} h_{0}$ is the line tension for the uncompressed brush and $F_{0} \sim \sigma N \sigma^{\frac{1}{\nu(d-1)}}$.

\section{FINITE SIZE EFFECTS}

In a semi-infinite brush, edge effects penetrate over the distance $\xi \sim h_{0}\left(h_{0} / h\right)^{1 / 2}$; finite size effects thus become important when the lateral extension of the brush becomes comparable to $\xi$. To be more specific, let us

\footnotetext{
${ }^{1}$ For finite $L$, a case considered shortly, there is indeed a minimum and a maximum, the latter being unphysical, here both extrema degenerate in an inflexion point
} 
take a stripe of width $2 L$, extending between $x=-L$ and $x=+L$, uniformly grafted with a density $\sigma$. The simple box model can again be solved exactly. The functional minimization of the free energy with respect to the splay $u(x)$ leads to the same Euler-Lagrange equation as in the semi-infinite case and the first integral given by eq.(5) still holds. However, the integration constant $C$ is no longer $1 / 4 \xi^{2}$. The integration constants are fixed by the requirements of vanishing splay at the stripe center $u(0)=0$ and of minimal line tension. After integration of eq.(5), the splay is then found to obey:

$$
\begin{aligned}
4 \tilde{x} & =-2 \tilde{u}+\operatorname{arcsinh}(\tilde{u} \delta) \\
\text { where: } \delta & =\frac{\tilde{u}_{L}}{\sinh \left(4 L / \xi+2 \tilde{u}_{L}\right)} \\
\text { with: } \frac{2 L}{\xi} & =\frac{1}{2} \operatorname{arctanh} 2 \tilde{u}_{L}-\tilde{u}_{L}
\end{aligned}
$$

For $L \gg \xi$ the semi-infinite brush result is recovered. At low values of $L / \xi$ however the reduced splay at the border vanishes as a power law $\tilde{u}_{L}=(3 L / 2 \xi)^{1 / 3}$. Thus in the strongly compressed bush, half of the chains of the stripe extend on either side in a brush of height $\sim N(\sigma L / h)^{1 / 3}$, as shown in Figure 4.

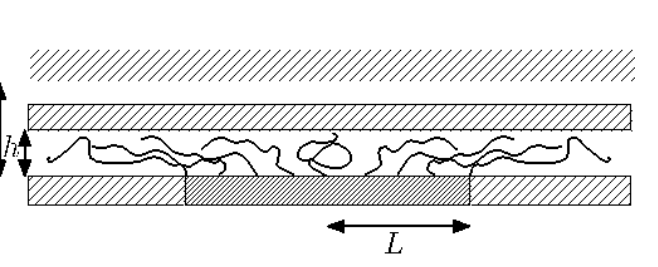

FIG. 4. Finite size effects : the grafted stripe. At high compression the penetration depth is bound by the half-size $L$. On either side, half of the chains build a brush parallel to the grafting surface at density $\sigma L / h$.

The scaling form for $u(L)=u_{L}$ is given by :

$$
\begin{aligned}
& u(L)=\xi \varphi(L / \xi) \\
& \text { with: } \quad \lim _{x \rightarrow \infty} \varphi(x)=1 / 2 \\
& \text { and } \varphi(x) \sim(3 x / 2)^{1 / 3} \quad(x \ll 1) .
\end{aligned}
$$

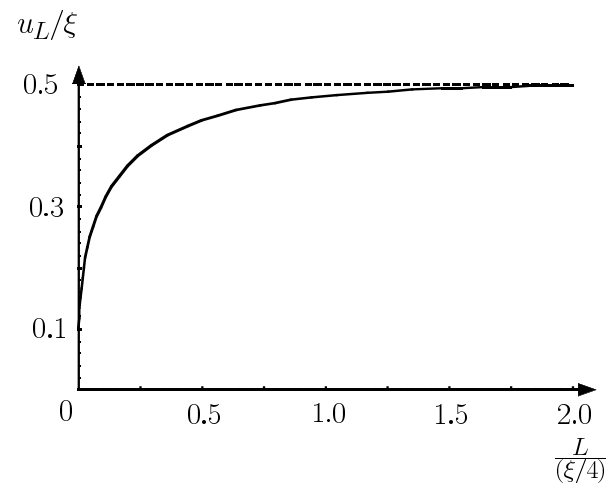

FIG. 5. Scaling of the splay at the border (stripe) : the reduced splay at the border $u_{L} / \xi$ is plotted against the reduced half-width $L /(\xi / 4)$ (we choose $\xi / 4$ as unit width because it is the decay length of the splay).

The complete scaling function, as obtained from eq.(11) is plotted in Figure 5. Assuming excluded volume statistics we obtain instead $\varphi(x) \sim x^{\frac{1-\nu}{\nu(d-1)}}$ for $x \ll 1$.

During compression, the layer evolves from a brush with chains oriented perpendicular to the grafting surface to a brush with chains oriented almost parallel to the surface. This entails a qualitative change in the force. For $L / \xi \ll 1$, the free energy is that of a brush parallel to the surface $\sim N h(\sigma L / h)^{5 / 3}$ and accordingly $f \sim N(\sigma L / h)^{5 / 3}$.

The explicit free energy can be computed from eq.(11)

$$
F=k_{\mathrm{B}} T \frac{\sigma L L_{y}}{N a^{2}}\left(-\frac{u_{L}^{3}}{6 L}+\frac{\xi^{2}}{4}+h^{2}\right),
$$

and shows how the "edge" term (first term) cancels against the osmotic term in the absence of splay (second term) to leading order at high compression. After derivation with respect to the brush height $h$ we obtain the force,

$$
f=k_{\mathrm{B}} T \frac{L L_{y} \sigma \xi^{2}}{4 N a^{2} h}\left(\frac{3}{2}-\frac{\tilde{u}_{L}^{3}}{L / \xi}-2 \tilde{u}_{L}^{2}-8 \frac{h^{2}}{\xi^{2}}\right) .
$$

At large width, the edge effects only contribute a $\xi / L$ edge correction. For small width $(L / \xi \ll 1)$, the force scales as $f \sim f_{\text {osm }}(L / \xi)^{2 / 3}$; here $f_{\text {osm }} \simeq k_{\mathrm{B}} T \frac{L L_{y} \sigma \xi^{2}}{4 N a^{2} h}$ is the osmotic force in the absence of any edge effect. The pressure on the layer increases as $L^{2 / 3}$. Pressure versus distance profiles are shown in Figure 6. The pressure unit is the osmotic pressure in the absence of any splay.

If the chains have excluded volume statistics, we can still consider qualitatively that a brush parallel to the grafting surface is formed at high compression. The free energy per unit area now scales as $\sim \sigma_{\text {eff }} N \sigma_{\text {eff }}^{\frac{1}{\nu(d-1)}}$ with $\sigma_{\text {eff }}=\sigma L / h$. The total free energy takes the simple form $F(L) \sim L L_{y} F_{\infty}(L / \xi)^{\frac{1}{\nu(d-1)}}(L \ll \xi)$, the force just scales as $f \sim F(L) / h$. 


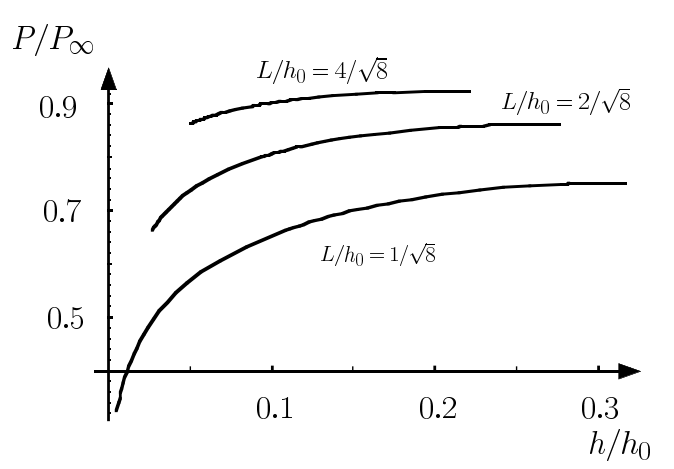

FIG. 6. Pressure versus height profiles (stripe). The pressure exerted to confine the brush (force divided by the grafted surface) as a function of the brush height. $P_{\infty}$ is the osmotic pressure exerted by an infinite brush of the same height (at the heights considered the elastic contribution is almost negligible), $h_{0}$ is the free brush height.

We now briefly consider a brush grafted on a finite disk of radius $R$, Figure 7. For a large disk $(R \gg \xi)$ the curvature has no effect and the splay at the border $u(R)$ is of order $\xi$.

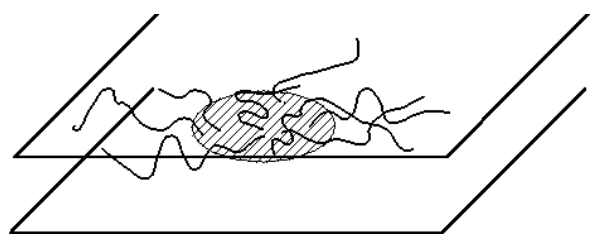

FIG. 7. The grafted disk. At high compression, the polymers escape laterally and form a cylindrical brush.

In general we anticipate a scaling form: $u(R)=\xi \varphi(R / \xi)$. For small disks, we expect the formation of a cylindrical brush that involves all grafted polymers. It is easily checked that most of the monomers belong to the corona extending outside the grafted disk. The local correlation length $\xi_{\phi}$ at a distance $r$ from the center follows from a Daoud and Cotton [1] type of argument, $\xi_{\phi}^{2} \sim \frac{r h}{\sigma R^{2}}$. The monomer concentration at a distance $r$ from the disk center is $c(r) \simeq\left(\frac{\sigma R^{2}}{r h}\right)^{3 \nu-1 /(2 \nu)}$. The conservation of monomers then leads to:

$$
u(R) \sim \xi\left(\frac{R}{\xi}\right)^{\frac{2(1-\nu)}{1+\nu}} \quad(R / \xi \ll 1)
$$

This holds at not too high compressions, as long as $\xi_{\phi}(R)<h$ otherwise a dilute corona is build beyond $r_{\text {dil }}$ given by $\xi_{\phi}\left(r_{\text {dil }}\right)=h$ where the polymers are constrained by the plates rather than by the other polymers [12]. In the regime where the polymers are semi-dilute through the whole corona, the free energy and the force obey the simple scaling form :

$$
F(R) \sim F_{\infty} R^{2}\left(\frac{R}{\xi}\right)^{\frac{2}{1+\nu}} \quad f(R) \sim F(R) / h
$$

and the pressure increases faster with the radius of the disk than with the width of the stripe, mainly because the polymer can escape in two dimensions for a disk and only in one dimension for a stripe (note that a formal increase in the dimension of space does not add any escape dimension for the polymer but only increases the longitudinal dimension of the stripe).

\section{CONCLUSION}

We have presented a study of the compression of finite size and semi-infinite brushes based on a Flory-type of calculation and scaling arguments. Chains close to the border relax some of their excluded volume interaction by creating a marked outward splay, which decreases towards the center of the brush.

For semi-infinite brushes, the very tail of the splay profile decays exponentially over a length $\sim \xi$. The penetration depth of the edge effects $\xi$ is much larger than both the natural brush height $h_{0}$ and the imposed height $h$; it is given by $\xi \sim h_{0}\left(h_{0} / h\right)^{\frac{1-\nu}{3 \nu-1}}$, and it is found to be the only relevant length scale. This means that chains grafted within the penetration depth $\xi$ form a brush almost parallel to the grafting surface of extension $\sim \xi$; this in turn is sufficient to determine the length $\xi$. The relaxation of the confinement constraint at the edge entails an effective negative line tension $\tau \sim-F_{\infty} \xi$ where $F_{\infty}$ is the free energy per unit area in an infinite brush.

Finite size effects occur when the lateral size $S$ of the layer (width $\mathrm{L}$ of a grafted stripe, radius $\mathrm{R}$ of a grafted disk) becomes of order $\xi$. The splay $u_{S}$ at the border is weaker than in the semi-infinite case : $u_{S} \sim \xi \varphi(S / \xi)$ with $\varphi(x)$ of order one for large argument and $\varphi(x) \propto x^{\alpha}$ for small arguments. The exponent $\alpha$ can be obtained from simple arguments: at high compression, all chains are collected to build a brush parallel to the grafting surfaces. The value obtained for a stripe $\alpha=\frac{1-\nu}{2 \nu}$ is slightly smaller than that obtained for a disk $\alpha=\frac{2(1-\nu)}{1+\nu}$.

As the brush (at least partially) escapes laterally during compression, the pressure $P$ exerted to confine the layer (evaluated with respect to the fixed grafted surface) increases with the brush size. More precisely, $P \sim P_{\infty}\left(\frac{S}{\xi}\right)^{\beta}$ where the value $\beta=1 /(2 \nu)$ for a stripe is smaller than that for a disk $\beta=2 /(1+\nu)$.

The layer is softer against compression with increasing border length: for a given grafted area, this means that layers divided in many small pieces, collections of stripes, or layers with holes are softer than compact layers. If the border lines are curved, positive curvatures (with the center of curvature located on the layer side) also make the brush softer. Rühe and coworkers synthesize grafted layers with controlled in plane structures. They are able 
to obtain long grafted chains by in situ radicalar polymerization on surfaces where lines seeded with (or free of) initiators are drawn via interference patterns. Those systems should be excellent model systems to study the mechanical response to compression.

The (weaker) negative effective line tension for unconstrained brushes has been used by Raphaël and de Gennes [6] to explain the formation of ribbon-shaped or disk-shaped aggregates in flexible(soluble)/rigid(insoluble)/flexible(soluble) triblock copolymers. One would expect the larger line tension in constrained systems to increase the length of the border line by formation of holes or cutlines.

When a diblock copolymer lamella (or stack of lamellas) is compressed, if the compression is released the lamellar period has to increase back to its equilibrium value. The characteristic time for an overall relaxation diverges with some (almost) macroscopic size. We thus expect relaxation to occur via the formation of defects (cuts and holes). A microscopic model such as the one developed here is needed to study the formation of these defects and their subsequent healing.

A related topic is the formation of stable defects close to a phase boundary in amphiphile/water systems [13]. A model very similar to the one presented here based on polymer physics could provide some molecular expressions for phenomenological quantities used in surfactant physics.

Microgels may spread on an adsorbing surface at the expense of elastic energy [14]. This can be seen as a compromise between surface tension and confinement. Confinement of the microgel starting from the swollen $c^{*}$ state can be studied in a way very similar to the study of polymer brushes presented here. To be specific take a piece of microgel of constant thickness irreversibly pasted on a flat solid by one face and adsorbing onto a parallel substrate by the opposite face. A very naive model for the gel is a perfect cubic gel that deforms upon confinement as the brush with the extra chain segments (crosslinking the brush chains) remaining parallel to the substrate. A length scale $\xi$ for the penetration of edge effects can again be defined as $\xi \sim h_{0}\left(h_{0} / h\right)^{1 / 2}$ where $h_{0}$ is the initial swelling equilibrium height. At the level of scaling arguments, balancing surface tension and confinement energy leads to the spreading condition (contact surface much larger than grafting surface), $\gamma>\sigma^{4 / 3} L^{2} / h_{0}$ in the case of a stripe (using mean-field exponents). The same spreading condition is obtained for the brush, however it is not clear whether the simple model used in this paper to describe the edge is suitable in this case.

\section{ACKNOWLEDGEMENT}

TAV greatfully acknowledges the kind hospitality of the Insitut Charles Sadron (CRM-CNRS) and the Laboratoire Européen Associé for financal support.

[1] D. Napper, Polymer stabilization of colloidal dispersions (Academic press, London, 1983).

[2] A. Halperin, M. Tirrell, and T. Lodge, Adv. Polym. Sci. 100, 31 (1992).

[3] S. Milner, J. Polym. Sci. B 32, 2743 (1994).

[4] S. Alexander, J. Physique 38, 983 (1977).

[5] P.-G. de Gennes, Scaling Concepts in Polymer Physics (Cornell University Press, Ithaca, N.Y., 1991).

[6] E. Raphael and P. de Gennes, Makromolekulare Chemie, Macromolecular Symposia 62, 1 (1992).

[7] A. Semenov, JETP 61, 733 (1985).

[8] S. Milner, T. Witten, and M. Cates, Macromol. 21, 2610 (1988).

[9] G. Fredrickson, A. Ajdari, L. Leibler, and J. Carton, Macromol. 25, 28820 (1992).

[10] H. Xi and S. Milner, Macromol. 29, 4772 (1996).

[11] M. Daoud and J.-P. Cotton, J. Physique 43, 531 (1982).

[12] A. Halperin and J. Joanny, J. Phys. II 1, 623 (1991).

[13] C. Bagdassarian, D. Roux, A. Ben-Shaul, and W. Gelbart, J. Chem. Phys. 94, 3030 (1991).

[14] T. A. Vilgis and M. Stapper, Eur. Phys. J. B 1, 69 (1998). 